\title{
Second Law Analysis for Third-Grade Fluid with Variable Properties
}

\author{
Samuel O. Adesanya \\ Department of Mathematical Sciences, Redeemer's University, Redemption City, PMB 2011, Sagamu, Ogun State, Nigeria \\ Correspondence should be addressed to Samuel O. Adesanya; adesanyaolumide@yahoo.com
}

Received 1 July 2014; Accepted 10 August 2014; Published 8 September 2014

Academic Editor: Pedro Jorge Martins Coelho

Copyright (C) 2014 Samuel O. Adesanya. This is an open access article distributed under the Creative Commons Attribution License, which permits unrestricted use, distribution, and reproduction in any medium, provided the original work is properly cited.

\begin{abstract}
This paper investigates the entropy generation in a third-grade fluid flow with variable properties through a channel. Approximate solutions to the nonlinear boundary-value problem are obtained using Adomian decomposition method (ADM). Variation of important parameters on the fluid velocity, temperature distribution, entropy generation and irreversibility ratio are presented graphically and discussed.
\end{abstract}

\section{Introduction}

Over the past few decades, there has been a tremendous increase in the study of heat transfer to viscous fluids due to its numerous applications in many industrial and engineering processes. As postulated in the second law of thermodynamics, the study could give insight into the thermal performance of the system by improving energy that is available for work $[1,2]$.

In the class of third-grade fluid, quite a lot has been done in recent times on entropy generation by assuming a constant thermal conductivity. For instance, Pakdemirli and Yilbas investigated the entropy generation in the flow of third-grade fluid through a pipe with constant viscosity in [3] while the temperature dependent viscosity has been investigated in [4] by using Vogel model. We refer interested readers to [514] for more interesting results on third-grade fluid flow. Moreover, Makinde and Aziz [15] presented the second law analysis of a pressure-driven temperature dependent fluid flow with asymmetry at the walls. Kahraman and Yürüsoy [16] examined the entropy generation due to non-Newtonian fluid flow in an annular pipe with relative rotation using a third-grade fluid model while Chauhan and Kumar [17] presented a non-Newtonian third-grade fluid flow in an annulus partially filled by a porous medium of very small permeability and many more results on entropy generation in literature.
Surprisingly, in spite of the enormous amount of work done on the entropy generation, it is observed that not much has been done on the exergy analysis of third-grade fluid flow with variable thermal conductivity and internal heat generation. As shown by Hayat et al. [18], these variations can significantly affect the flow field. Hence a more accurate result could be obtained by taking these variations into consideration.

Therefore, the specific objective of this paper is to investigate the entropy generation in the flow of third-grade fluid channel flow with temperature dependent properties. The problem under discussion is strongly nonlinear boundary valued problem. Approximate solution will be obtained using Adomian decomposition method as presented by Siddiqui et al. [19] for the third-grade fluid flow. Therefore, in the absence of internal heat generation and neglecting variations with temperature, the result obtained in [19] will be fully recovered. The seminumerical method has also been used to obtain approximate solution to different fluid flow problems in [20-22]. To the best of the author's knowledge, the study reported here on entropy generation in a heat generating third-grade fluid flow with variable properties has not been undertaken in literature.

The rest of the paper is organized as follows. In the following section, the mathematical analysis of the flow is presented. Section 3 describes the method of obtaining the approximate solution of the nonlinear problem. In Section 4, 


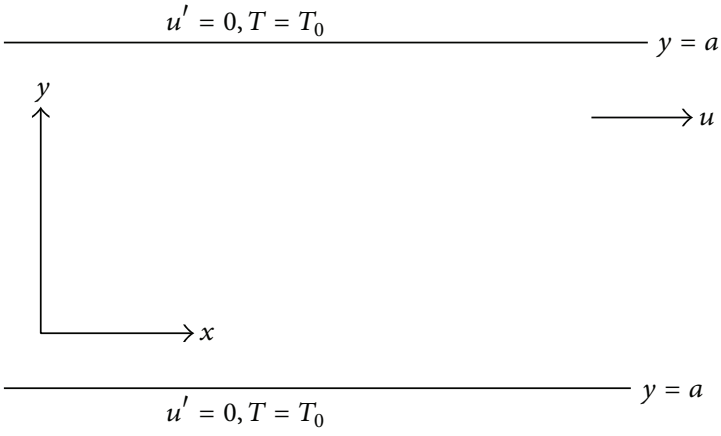

FIGURE 1: Channel geometry.

results are presented and discussed before the paper is concluded in Section 5.

\section{Mathematical Analysis}

Consider the steady flow of a third-grade fluid with variable viscosity and thermal conductivity through infinite parallel plates of distance $2 a$ apart as shown in Figure 1.

The fluid is assumed to be reactive and internal heat generation is assumed to be a linear function of temperature. Then the governing equations for the fully developed flow can be written as $[15,18]$

$$
\begin{aligned}
0= & -\frac{d P}{d x}+\frac{d}{d y^{\prime}}\left(\mu^{\prime} \frac{d u^{\prime}}{d y^{\prime}}\right)+6 \beta_{3} \frac{d^{2} u^{\prime}}{d y^{\prime 2}}\left(\frac{d u^{\prime}}{d y^{\prime}}\right)^{2}, \\
0= & \frac{d}{d y^{\prime}}\left(k^{\prime} \frac{d T}{d y^{\prime}}\right)+\mu^{\prime}\left(\frac{d u^{\prime}}{d y^{\prime}}\right)^{2} \\
& +2 \beta_{3}\left(\frac{d u^{\prime}}{d y^{\prime}}\right)^{4}+Q_{0}\left(T-T_{0}\right),
\end{aligned}
$$

and the entropy generation is given as

$$
E_{G}=\frac{k^{\prime}}{T_{0}^{2}}\left(\frac{d T}{d y^{\prime}}\right)^{2}+\frac{1}{T_{0}}\left(\frac{d u^{\prime}}{d y^{\prime}}\right)^{2}\left(\mu^{\prime}+2 \beta_{3}\left(\frac{d u^{\prime}}{d y^{\prime}}\right)^{2}\right)
$$

with appropriate boundary conditions

$$
\begin{aligned}
& u^{\prime}=0, \quad T=T_{0}, \quad \text { on } y^{\prime}=a, \\
& \frac{d u^{\prime}}{d y^{\prime}}=0=\frac{d T}{d y^{\prime}}, \quad \text { on } y^{\prime}=0 ;
\end{aligned}
$$

the temperature dependent viscosity and thermal conductivity take the form

$$
\begin{aligned}
\mu^{\prime} & =\mu-\eta_{0}\left(T-T_{0}\right), \\
k^{\prime} & =k+\eta_{1}\left(T-T_{0}\right),
\end{aligned}
$$

where $u^{\prime}$ is the fluid velocity, $T$ is the fluid temperature, $P$ is the fluid pressure, $a$ is the channel half width, $\beta_{3}$ is the material coefficient, $k$ is the thermal conductivity, $\mu^{\prime}$ is the dynamic viscosity, $\eta_{0,1}$ are the viscosity and thermal conductivity variation parameters, $Q_{0}$ is the heat generated internally, $T_{0}$ and $T_{1}$ are referenced fluid temperature and $E_{G}$ is the entropy generation.

Introducing the following dimensionless parameters and variables:

$$
\begin{gathered}
y=\frac{y^{\prime}}{a}, \quad u=\frac{u^{\prime}}{U}, \quad \gamma=\frac{\beta_{3} U^{2}}{a^{2} \mu}, \\
\theta=\frac{T-T_{0}}{T_{1}-T_{0}}, \quad \alpha=\frac{\eta_{0}\left(T_{1}-T_{0}\right)}{\mu}, \quad \Omega=\frac{T_{1}-T_{0}}{T_{0}}, \\
G=-\frac{a^{2}}{\mu U} \frac{d P}{d x}, \quad \delta=\frac{Q_{0} a^{2}}{k}, \quad B r=\frac{\mu U^{2}}{k_{0}\left(T_{1}-T_{0}\right)}, \\
\lambda=\frac{\eta_{1}\left(T_{1}-T_{0}\right)}{k}, \quad N_{s}=\frac{T_{0}^{2} a^{2} E_{G}}{k\left(T_{1}-T_{0}\right)^{2}},
\end{gathered}
$$

we get the following dimensionless equations together with appropriate boundary conditions:

$$
\begin{gathered}
\frac{d^{2} u}{d y^{2}}=\alpha\left[\theta \frac{d^{2} u}{d y^{2}}+\frac{d u}{d y} \frac{d \theta}{d y}\right]-6 \gamma \frac{d^{2} u}{d y^{2}}\left(\frac{d u}{d y}\right)^{2}-G \\
u( \pm 1)=0 \\
\frac{d^{2} \theta}{d y^{2}}=-\lambda\left[\left(\frac{d \theta}{d y}\right)^{2}+\theta \frac{d^{2} \theta}{d y^{2}}\right] \\
-\operatorname{Br}\left(\frac{d u}{d y}\right)^{2}\left\{(1-\alpha \theta)+2 \gamma\left(\frac{d u}{d y}\right)^{2}\right\}+\delta \theta \\
N_{s}=(1+\lambda \theta)\left(\frac{d \theta}{d y}\right)^{2}+\frac{\operatorname{Br}}{\Omega}\left(\frac{d u}{d y}\right)^{2}\left\{(1-\alpha \theta)+2 \gamma\left(\frac{d u}{d y}\right)^{2}\right\} .
\end{gathered}
$$

If we define

$$
\begin{aligned}
& N_{1}=(1+\lambda \theta)\left(\frac{d \theta}{d y}\right)^{2}, \\
& N_{2}=\frac{\operatorname{Br}}{\Omega}\left(\frac{d u}{d y}\right)^{2}\left\{(1-\alpha \theta)+2 \gamma\left(\frac{d u}{d y}\right)^{2}\right\},
\end{aligned}
$$

then the irreversibility ratio denoted by Bejan number (Be) can be written as

$$
\mathrm{Be}=\frac{N_{1}}{N_{s}}=\frac{N_{1}}{N_{1}+N_{2}}=\frac{1}{1+\Phi}, \quad \Phi=\frac{N_{2}}{N_{1}},
$$

here $u$ is the dimensionless velocity, $\alpha$ is the viscosity variation parameter, $\gamma$ is the third-grade material effect, $G$ is the pressure gradient, $\theta$ is the dimensionless fluid temperature, $\lambda$ is the thermal conductivity variation parameter, $\mathrm{Br}$ is Brinkman number, $\delta$ is the internal heat generation parameter, $\Omega$ is the parameter that measures the temperature difference between the two heat reservoirs, $N_{s}$ is the dimensionless entropy generation rate, and $U$ represents the characteristic velocity. 
TABLE 1: Convergence result for $\gamma=0.2, \delta=1, \mathrm{Br}=0.4, \alpha=0.5=\lambda, y=0.1$.

\begin{tabular}{ccccc}
\hline$n$ & $u_{n}$ & $\sum_{n=0}^{m} u_{n}$ & $\theta_{n}$ & $\sum_{n=0}^{m} \theta_{n}$ \\
\hline 0 & 0.445252 & 0.445252 & 0.0507153 & 0.0507153 \\
1 & -0.000116788 & 0.445135 & -0.000256831 & 0.0504585 \\
2 & $-1.96106 \times 10^{-6}$ & 0.445134 & $6.56661 \times 10^{-6}$ & 0.0504650 \\
3 & $-2.58151 \times 10^{-8}$ & 0.445133 & $-1.93224 \times 10^{-7}$ & 0.0504648 \\
4 & $1.74273 \times 10^{-10}$ & 0.445133 & $5.79716 \times 10^{-9}$ & 0.0504648 \\
\hline
\end{tabular}

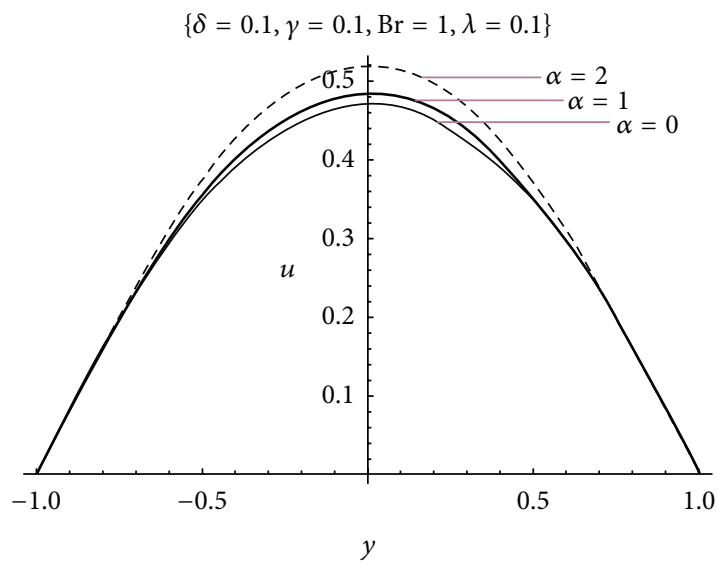

FIGURE 2: Effect of viscosity variation parameter on velocity profile.

\section{Adomian Decomposition Method of Solution}

The integral form of (6) can be written as

$$
\begin{aligned}
u(y)=a_{0}+\iint_{0}^{y}\{\alpha & {\left[\theta \frac{d^{2} u}{d Y^{2}}+\frac{d u}{d Y} \frac{d \theta}{d Y}\right] } \\
& \left.-6 \gamma \frac{d^{2} u}{d Y^{2}}\left(\frac{d u}{d Y}\right)^{2}-G\right\} d Y d Y,
\end{aligned}
$$

$\theta(y)$

$$
\begin{aligned}
=b_{0}-\iint_{0}^{y}\{\lambda & {\left[\left(\frac{d \theta}{d Y}\right)^{2}+\theta \frac{d^{2} \theta}{d Y^{2}}\right]+\operatorname{Br}\left(\frac{d u}{d Y}\right)^{2} } \\
\times & \left.\left\{(1-\alpha \theta)+2 \gamma\left(\frac{d u}{d Y}\right)^{2}\right\}+\delta \theta\right\} d Y d Y,
\end{aligned}
$$

where the constants $a_{0}$ and $b_{0}$ are to be determined later; the standard Adomian decomposition method [19-21] assumes series solutions in the form

$$
u=\sum_{n=0}^{\infty} u_{n}, \quad \theta=\sum_{n=0}^{\infty} \theta_{n}
$$

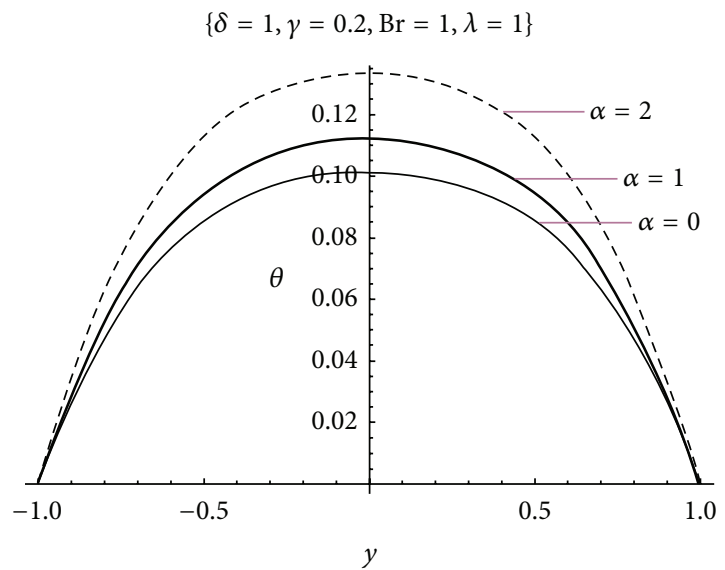

FIGURE 3: Effect of viscosity variation parameter on temperature profile.

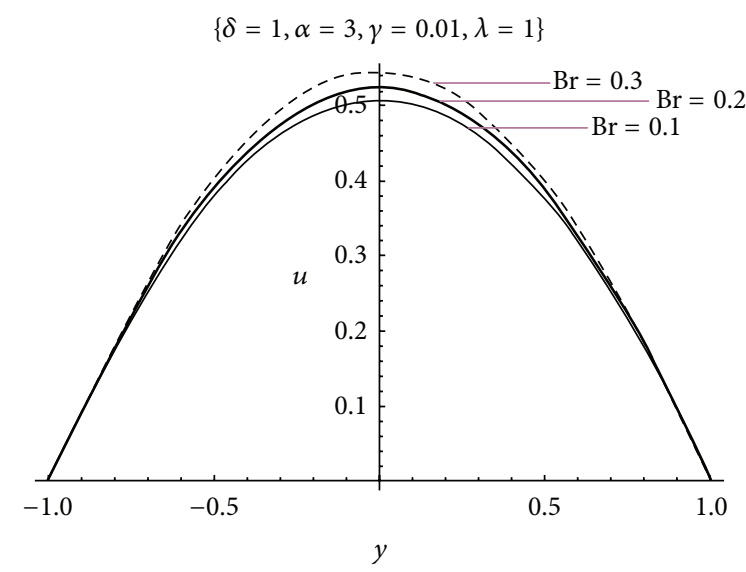

FIGURE 4: Effect of Brinkman number on velocity profile.

substituting (11) into the integral equations (10) yields the following recursive algorithm:

$$
\begin{gathered}
\theta_{0}(y)=b_{0}, \\
u_{0}(y)=a_{0}-\iint_{0}^{y}(G) d Y d Y, \\
u_{n+1}(y)=\iint_{0}^{y}\left\{\alpha A_{n}-6 \gamma B_{n}\right\} d Y d Y, \quad n \geq 1,
\end{gathered}
$$




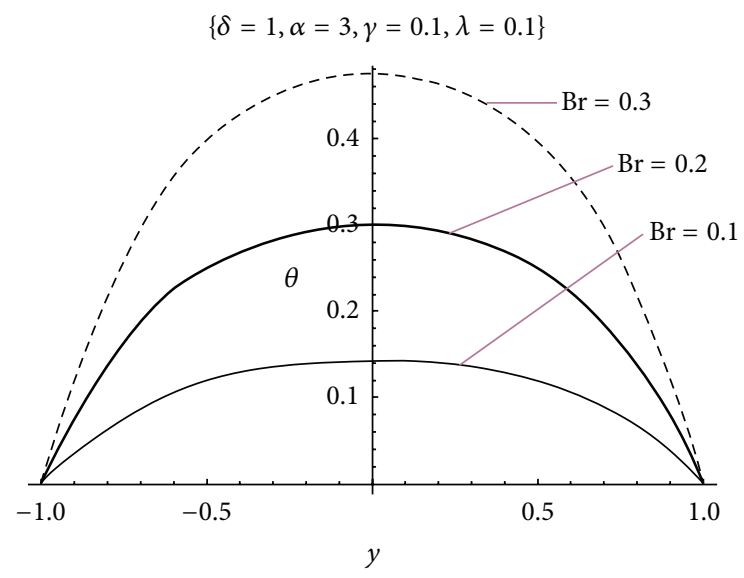

Figure 5: Effect of Brinkman number on temperature profile.

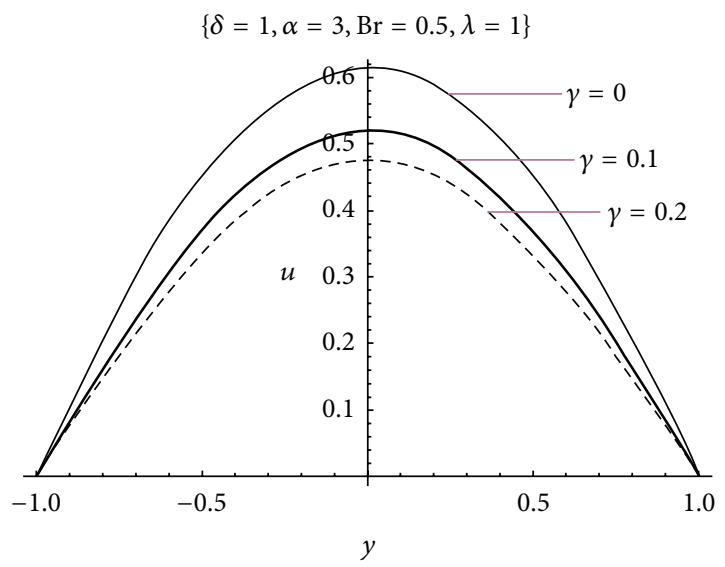

FIGURE 6: Effect of non-Newtonian material on velocity profile.

$$
\begin{array}{r}
\theta_{n+1}(y) \\
=-\iint_{0}^{y}\left[\lambda C_{n}+\operatorname{Br} F_{n}\left\{\left(1-\alpha \theta_{n}\right)+2 \gamma F_{n}\right\}+\delta \theta_{n}\right] d Y d Y ; \\
n \geq 1 ;
\end{array}
$$

the approximate solution is given by the partial sum

$$
\theta=\sum_{n=0}^{m} \theta_{n}, \quad u=\sum_{n=0}^{m} u_{n}
$$

where $m$ represent the truncation point. The nonlinear terms in (12) represented by

$$
\begin{gathered}
A_{n}=\frac{d u_{n}}{d Y} \frac{d \theta_{n}}{d Y}+\theta_{n} \frac{d^{2} u_{n}}{d Y^{2}}, \quad B_{n}=\frac{d^{2} u_{n}}{d Y^{2}}\left(\frac{d u_{n}}{d Y}\right)^{2}, \\
C_{n}=\left(\frac{d \theta_{n}}{d Y}\right)^{2}+\theta_{n} \frac{d^{2} \theta_{n}}{d Y^{2}}, \quad F_{n}=\left(\frac{d u_{n}}{d Y}\right)^{2}
\end{gathered}
$$

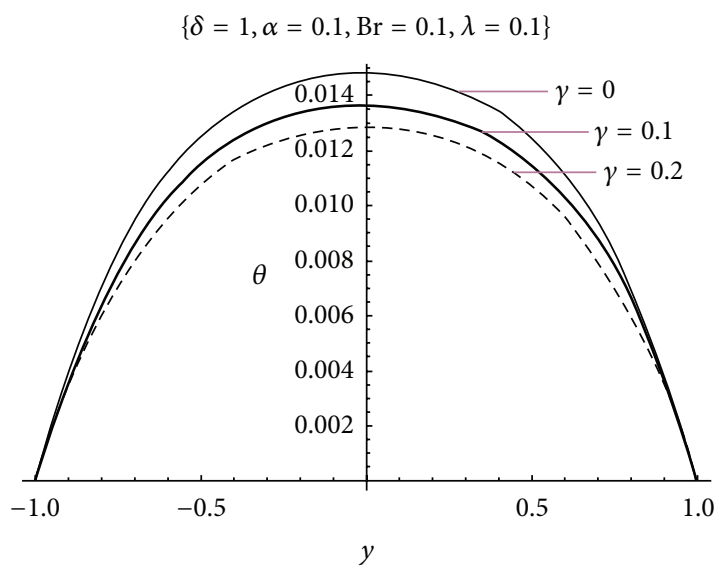

FIGURE 7: Effect of non-Newtonian material on temperature profile.

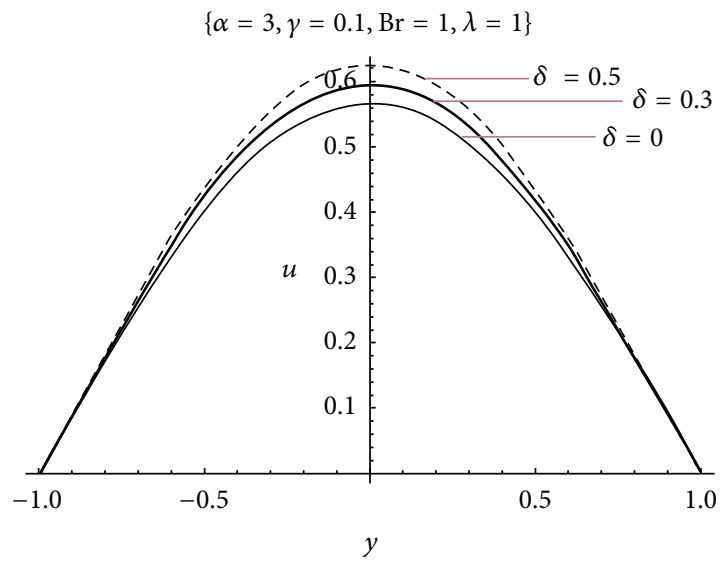

FIGURE 8: Effect of internal heat generation parameter on velocity profile.

are decomposed into Adomian polynomials as follows:

$$
\begin{aligned}
A_{0}= & \frac{d \theta_{0}}{d Y} \frac{d u_{0}}{d Y}+\theta_{0} \frac{d^{2} u_{0}}{d Y^{2}}, \\
A_{1}= & \frac{d \theta_{0}}{d Y} \frac{d u_{1}}{d Y}+\frac{d \theta_{1}}{d Y} \frac{d u_{0}}{d Y}+\theta_{1} \frac{d^{2} u_{0}}{d Y^{2}}+\theta_{0} \frac{d^{2} u_{1}}{d Y^{2}} \\
A_{2}= & \frac{d \theta_{0}}{d Y} \frac{d u_{2}}{d Y}+\frac{d \theta_{1}}{d Y} \frac{d u_{1}}{d Y}+\frac{d \theta_{2}}{d Y} \frac{d u_{0}}{d Y}+\theta_{2} \frac{d^{2} u_{0}}{d Y^{2}} \\
& +\theta_{1} \frac{d^{2} u_{1}}{d Y^{2}}+\theta_{0} \frac{d^{2} u_{2}}{d Y^{2}},
\end{aligned}
$$

similarly,

$$
\begin{aligned}
B_{0}= & \frac{d^{2} u_{0}}{d Y^{2}}\left(\frac{d u_{0}}{d Y}\right)^{2}, \\
B_{1}= & 2 \frac{d^{2} u_{0}}{d Y^{2}}\left(\frac{d u_{0}}{d Y}\right)\left(\frac{d u_{1}}{d Y}\right)+\frac{d^{2} u_{1}}{d Y^{2}}\left(\frac{d u_{0}}{d Y}\right)^{2}, \\
B_{2}= & \frac{d^{2} u_{0}}{d Y^{2}}\left(\frac{d u_{1}}{d Y}\right)^{2}+2 \frac{d^{2} u_{0}}{d Y^{2}}\left(\frac{d u_{0}}{d Y}\right)\left(\frac{d u_{2}}{d Y}\right) \\
& +2 \frac{d^{2} u_{1}}{d Y^{2}}\left(\frac{d u_{0}}{d Y}\right)\left(\frac{d u_{1}}{d Y}\right)+\frac{d^{2} u_{2}}{d Y^{2}}\left(\frac{d u_{0}}{d Y}\right)^{2},
\end{aligned}
$$




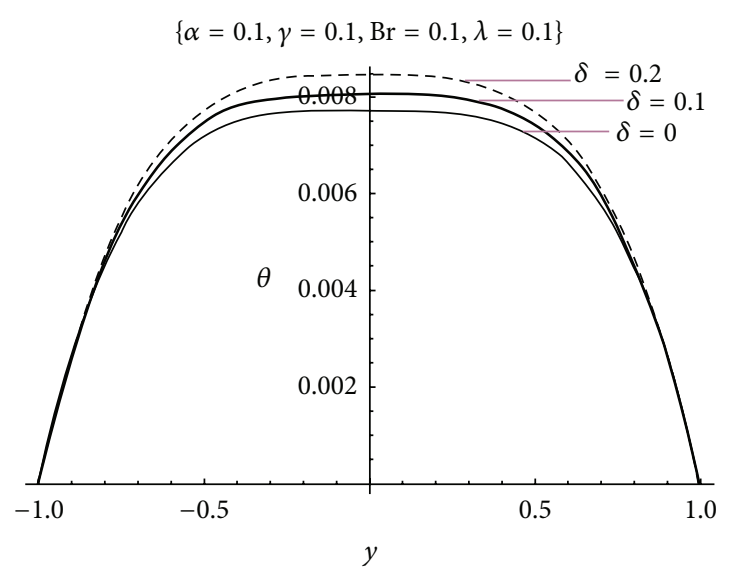

FIGURE 9: Effect of internal heat generation parameter on temperature profile.

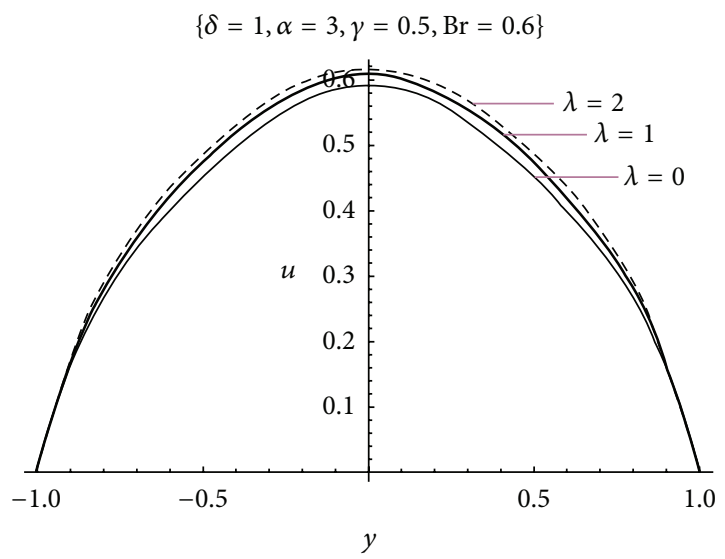

FIGURE 10: Effect of thermal conductivity variation parameter on velocity profile.

with

$$
\begin{aligned}
& F_{0}=\left(\frac{d u_{0}}{d Y}\right)^{2} \\
& F_{1}=2 \frac{d u_{0}}{d Y} \frac{d u_{1}}{d Y} \\
& F_{2}=2 \frac{d u_{0}}{d Y} \frac{d u_{2}}{d Y}+\left(\frac{d u_{1}}{d Y}\right)^{2}, \ldots \\
& C_{0}=\left(\frac{d \theta_{0}}{d Y}\right)^{2}+\theta \frac{d^{2} \theta_{0}}{d Y^{2}}, \\
& C_{1}=2 \frac{d \theta_{0}}{d Y} \frac{d \theta_{1}}{d Y}+\theta_{0} \frac{d^{2} \theta_{1}}{d Y^{2}}+\theta_{1} \frac{d^{2} \theta_{0}}{d Y^{2}}, \\
& C_{2}=2 \frac{d \theta_{0}}{d Y} \frac{d \theta_{2}}{d Y}+\left(\frac{d \theta_{1}}{d Y}\right)^{2}+\theta_{2} \frac{d^{2} \theta_{0}}{d Y^{2}}+\theta_{1} \frac{d^{2} \theta_{1}}{d Y^{2}}+\theta_{0} \frac{d^{2} \theta_{2}}{d Y^{2}}, \ldots
\end{aligned}
$$

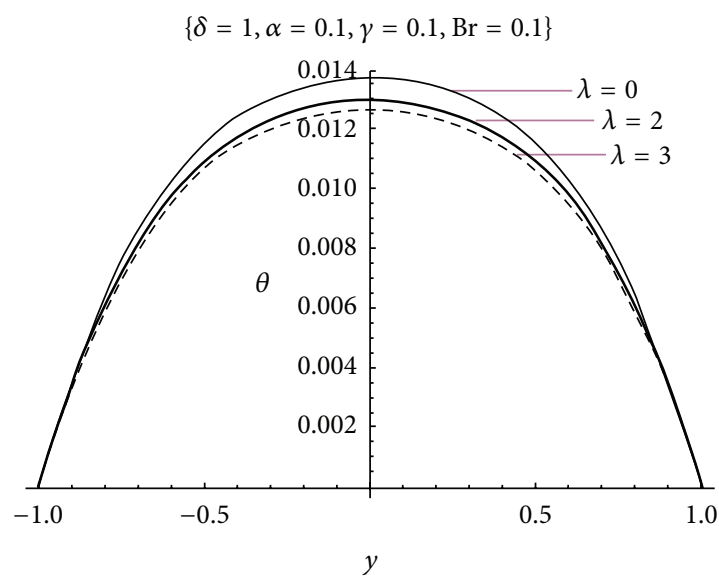

FIGURE 11: Effect of thermal conductivity variation parameter on temperature profile.

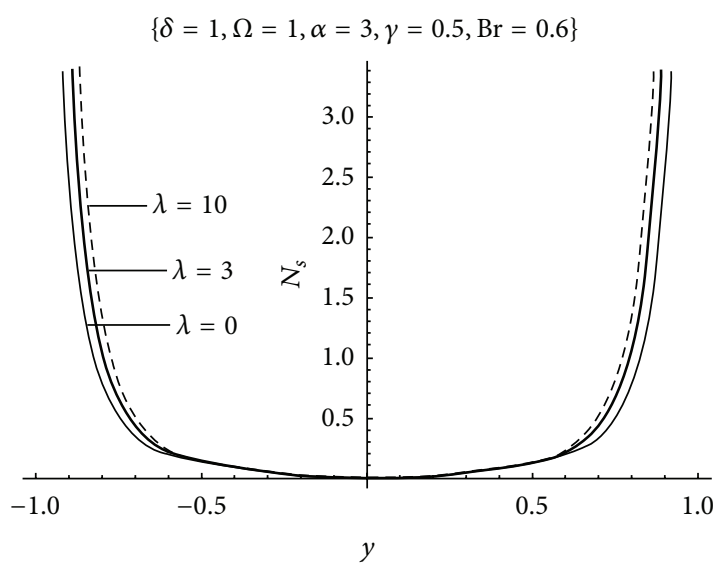

FIGURE 12: Effect of thermal conductivity variation parameter on entropy generation rate.

\section{Results and Discussion}

The major objective of this work is to investigate the effects of temperature dependent viscosity and thermal conductivity on heat generating third-grade fluid flow through a channel with isothermal temperature. Approximate solutions to the nonlinear boundary-value problem are obtained in the form of a rapidly convergent Adomian series solution. Table 1 confirmed that series solution is convergent and reliable. Figure 2 shows the velocity profile for variations in the viscosity variation parameter. As observed from the graph, an increase in the viscosity variation parameter enhances the flow. This is physically true due to the melting effect on the fluid viscosity. Similarly, Figure 3 shows that an increase in the viscosity variation parameter increases the temperature profile due to rise in the temperature difference within the channel. Figures 4 and 5 show the velocity and temperature profiles, respectively, for variation in Brinkman number. From the plots it is observed that an increase in the Brinkman number leads to an increase in both the fluid velocity and temperature due to rise in the kinetic energy 


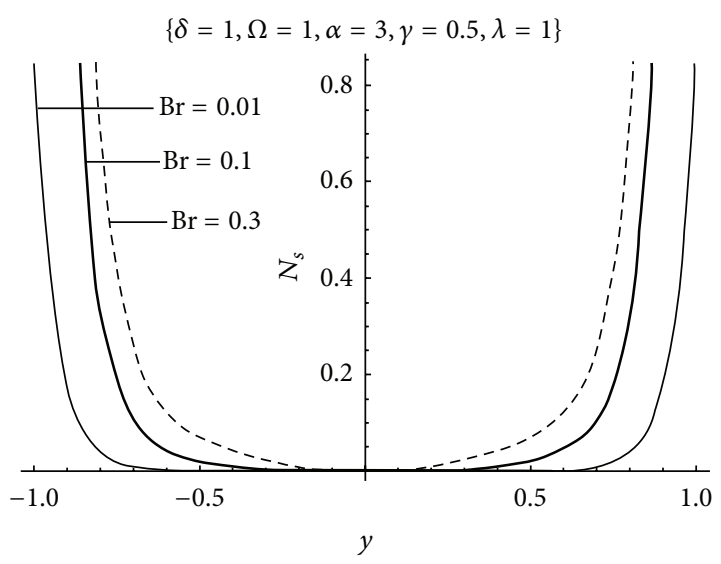

FIGURE 13: Effect of Brinkman number on entropy generation rate.

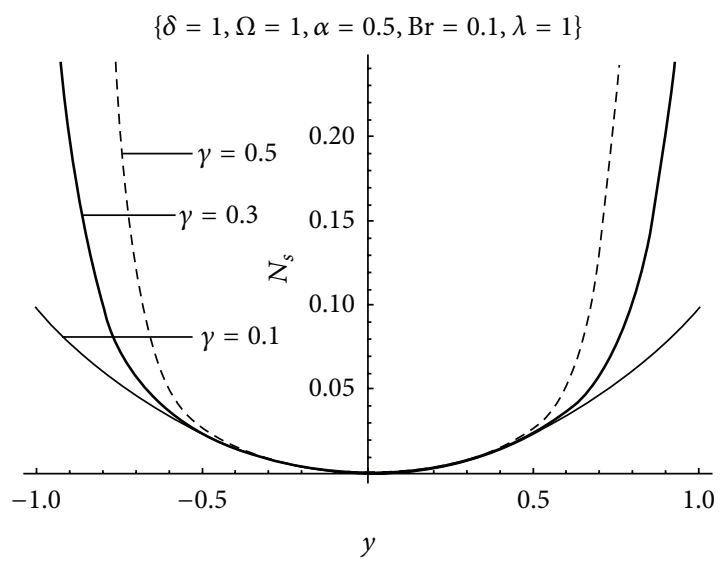

FIGURE 14: Effect of third-grade material effect on entropy generation rate.

within the moving fluid. Moreover, Figures 6 and 7 represent the effect of third-grade material effect on the velocity and temperature profiles; the result shows that an increase in the non-Newtonian material effect decreases both the velocity and temperature profiles due to fluid thickening. Figure 8 depicts the velocity profile for variations in internal heat generation parameter. The result shows that an increase in heat generation parameter increases the flow velocity due to rise in the concentration of the reacting fluid. However, as observed in Figure 9, an increase in the internal heat generation increases the temperature distribution within the channel. Figures 10 and 11 display the effect of variations in thermal conductivity on both the velocity and temperature distributions within the channel. As observed from Figure 10, an increase in the thermal conductivity variation parameter increases the velocity distribution within the channel. On the other hand, an increase in the thermal conductivity variation parameter implies a decrease in the thermal conductivity of the fluid. This ultimately decreases the fluid temperature as observed in Figure 11. As observed from the plots, Figures 12,13 , and 14 show that, in an increase in thermal

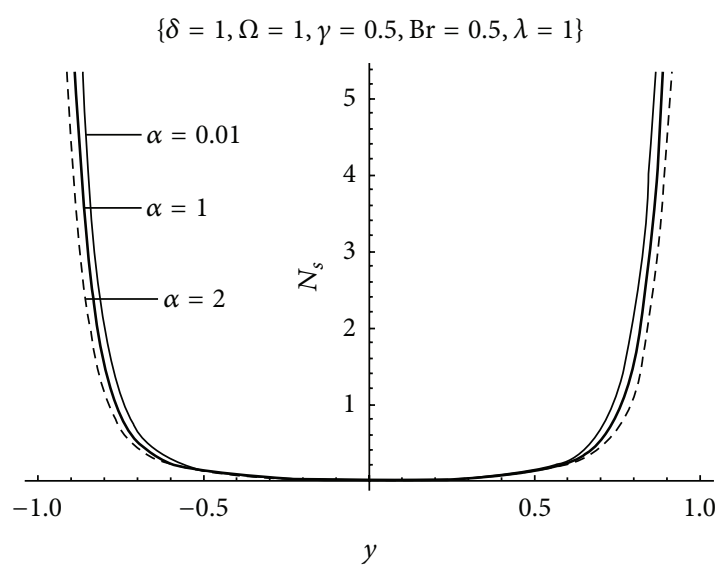

FIGURE 15: Effect of viscosity variation parameter on entropy generation rate.

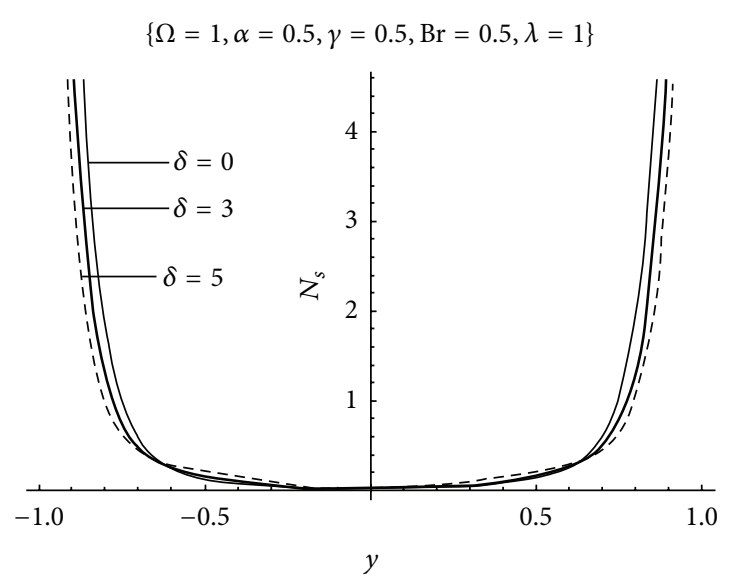

FIGURE 16: Effect of internal heat generation parameter on entropy generation rate.

conductivity variation parameter, Brinkman number and third-grade material effect enhance the entropy generation rate within the channel, while increase in viscosity variation parameter decreases the entropy generation rate within the channel as seen in Figure 15. In Figure 16, rise in the internal heat generation parameter is observed to increase entropy generation in the center line of the channel while entropy generation decreases at the walls. Moreover, Figures 17 and 18 shows that as the internal heat generation and Brinkman number increases heat transfer dominates over the fluid viscosity within the channel. Figure 19 represents the effect of viscosity variation parameter on the irreversibility ratio; from the plot it is observed that as the viscosity variation parameter increases, heat transfer dominates over the fluid viscosity at the walls whereas at the centerline of the channel fluid viscosity is observed to dominate over heat transfer. Finally, Figures 20 and 21 show that rise in increase in both thirdgrade material effect and thermal conductivity variation parameter shows that fluid viscosity dominates over the heat transfer within the channel. 


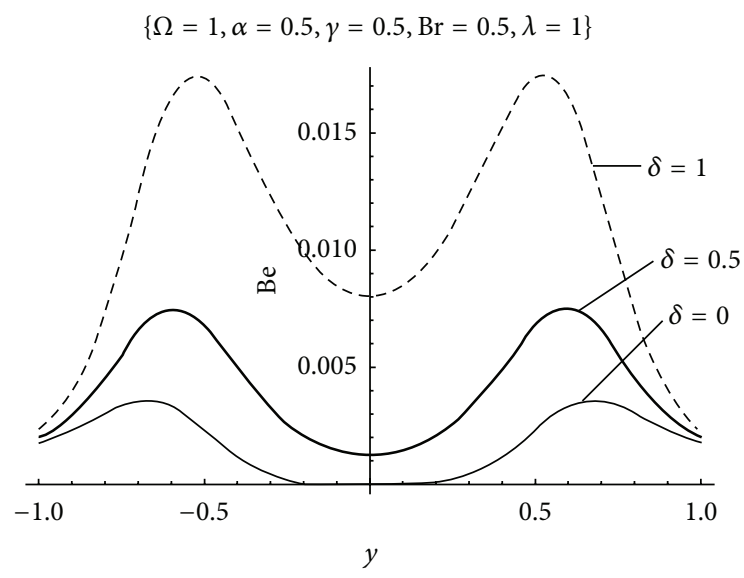

FIGURE 17: Effect of internal heat generation on the irreversibility ratio.

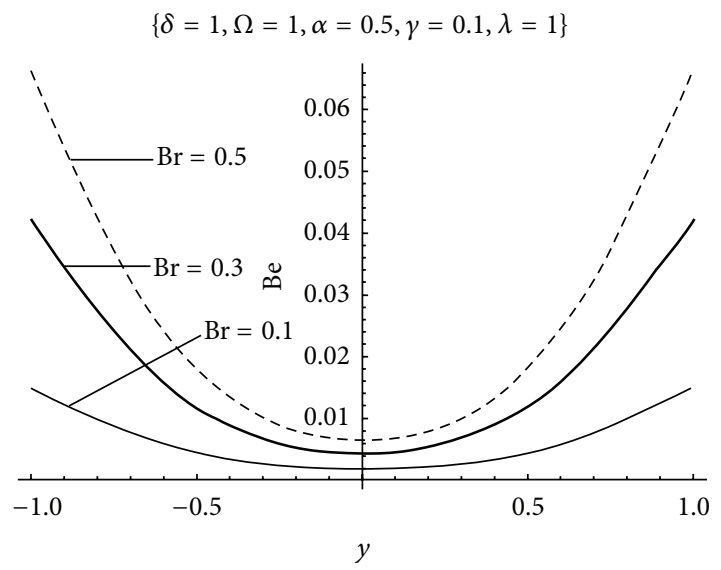

FIGURE 18: Effect of Brinkman number on the irreversibility ratio.

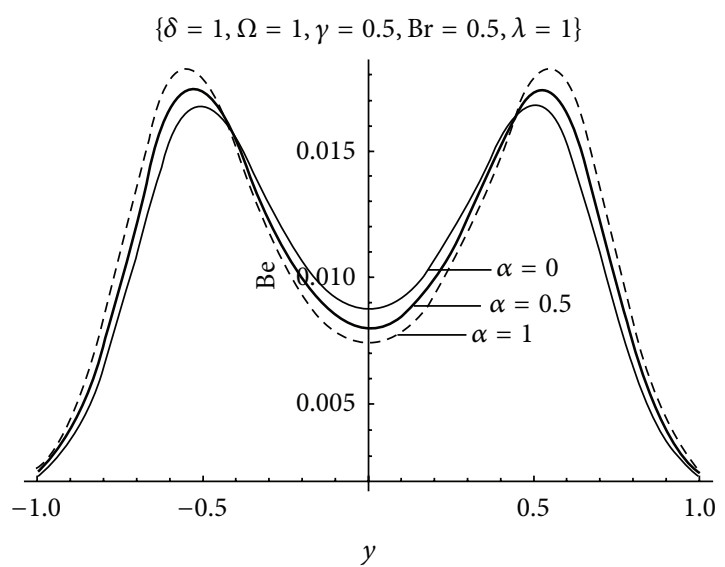

FIGURE 19: Effect of viscosity variation parameter on the irreversibility ratio.

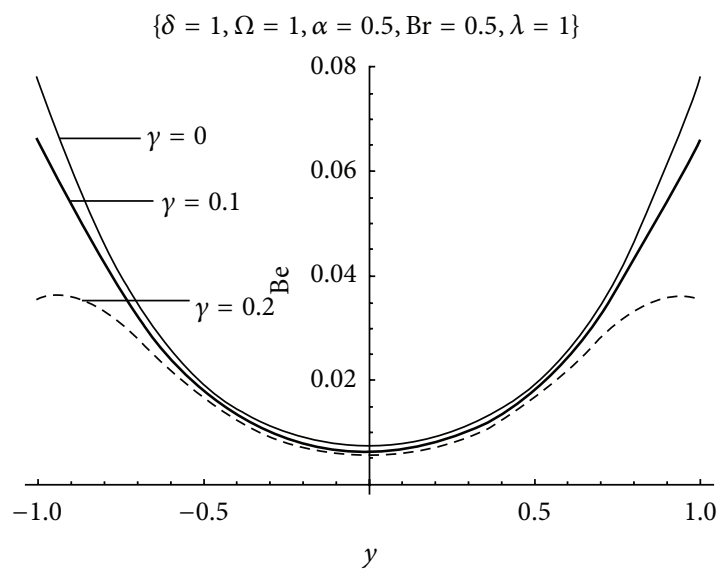

FIGURE 20: Effect of third-grade material effect on irreversibility ratio.

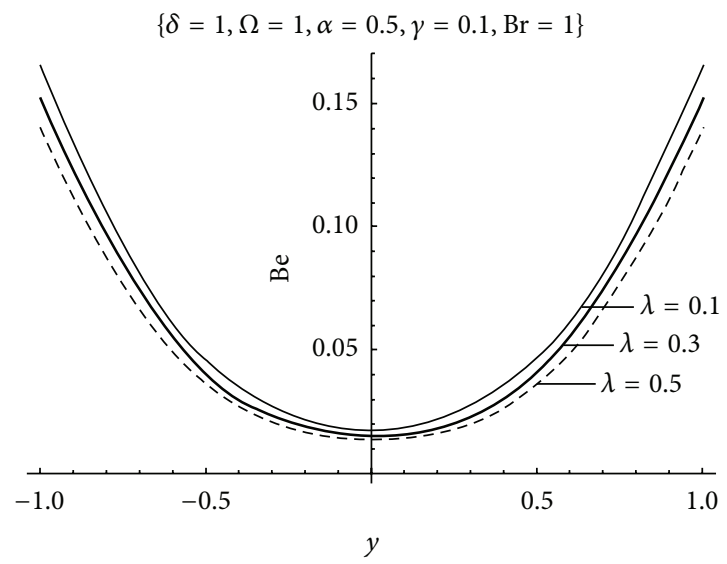

FIGURE 21: Effect of thermal conductivity variation parameter on irreversibility ratio.

\section{Conclusion}

In this paper, the entropy generation analysis for third-grade fluid flow with variable properties through a channel with uniform wall temperature is studied. In the limiting case, there is perfect agreement between the present result and that obtained in [19] when $\lambda=\delta=\alpha=0$. Summarily, the main contributions to knowledge from the computation are as follows.

(1) Rise in the viscosity variation parameter is observed to decrease the entropy generation rate within the channel. Moreover, in the middle of the channel, fluid viscosity is observed to dominate over heat transfer. A reverse behaviour is observed at the channel walls.

(2) An increase in the fluid thermal conductivity enhances entropy generation rate. Interestingly, it supports the dominance of fluid viscosity over the heat transfer within the channel.

(3) An increase in the internal heat generation parameter increases the entropy generation rate in the centreline of the channel only. 
One major area of future research is the flow behavior for large values of the thermal conductivity parameter. To achieve this further analysis will be required to determine the inherent thermal criticality.

\section{Conflict of Interests}

The author declares that there is no conflict of interests regarding the publication of this paper.

\section{References}

[1] S. O. Adesanya and O. D. Makinde, "Entropy generation in couple stress fluid flow through porous channel with fluid slippage," International Journal of Exergy. In press.

[2] S. O. Adesanya and O. D. Makinde, "Effects of couple stresses on entropy generation rate in a porous channel with convective heating," Computational and Applied Mathematics.

[3] M. Pakdemirli and B. S. Yilbas, "Entropy generation in a pipe due to non-Newtonian fluid flow: constant viscosity case," Sadhana, vol. 31, no. 1, pp. 21-29, 2006.

[4] M. Pakdemirli and B. S. Yilbas, "Entropy generation for pipe flow of a third grade fluid with Vogel model viscosity," International Journal of Non-Linear Mechanics, vol. 41, no. 3, pp. 432437, 2006.

[5] R. Ellahi, "A study on the convergence of series solution of nonNewtonian third grade fluid with variable viscosity: by means of homotopy analysis method," Advances in Mathematical Physics, vol. 2012, Article ID 634925, 11 pages, 2012.

[6] R. Ellahi, T. Hayat, and F. M. Mahomed, "Generalized couette flow of a third-grade fluid with slip: the exact solutions," Zeitschrift fur Naturforschung A, vol. 65, no. 12, pp. 1071-1076, 2010.

[7] R. Ellahi and A. Riaz, "Analytical solution for MHD flow in a third-grade fluid with variable viscosity," Mathematical and Computer Modelling, vol. 52, no. 9-10, pp. 1783-1793, 2010.

[8] R. Ellahi, T. Hayat, F. M. Mahomed, and S. Asghar, "Effects of slip on the non-linear flows of a third grade fluid," Nonlinear Analysis: Real World Applications, vol. 11, no. 1, pp. 139-146, 2010.

[9] S. Abbasbandy, T. Hayat, F. M. Mahomed, and R. Ellahi, "On comparison of exact and series solutions for thin film flow of a third-grade fluid," International Journal for Numerical Methods in Fluids, vol. 61, no. 9, pp. 987-994, 2009.

[10] R. Ellahi, P. D. Ariel, T. Hayat, and S. Asghar, "Effect of heat transfer on a third grade fluid in a flat channel," International Journal for Numerical Methods in Fluids, vol. 63, no. 7, pp. 847859, 2010.

[11] T. Hayat, R. Ellahi, and F. M. Mahomed, "The analytical solutions for magnetohydrodynamic flow of a third order fluid in a porous medium," Zeitschrift fur Naturforschung A Journal of Physical Sciences, vol. 64, no. 9-10, pp. 531-539, 2009.

[12] T. Hayat, R. Ellahi, and F. M. Mahomed, "Exact solutions for thin film flow of a third grade fluid down an inclined plane," Chaos, Solitons \& Fractals, vol. 38, no. 5, pp. 1336-1341, 2008.

[13] R. Ellahi and S. Afzal, "Effects of variable viscosity in a third grade fluid with porous medium: an analytical solution," Communications in Nonlinear Science and Numerical Simulation, vol. 14, no. 5, pp. 2056-2072, 2009.

[14] R. Ellahi, "The thermodynamics, stability, applications and techniques of differential type: a review," Reviews in Theoretical Science, vol. 2, pp. 116-123, 2014.
[15] O. D. Makinde and A. Aziz, "Second law analysis for a variable viscosity plane Poiseuille flow with asymmetric convective cooling," Computers \& Mathematics with Applications, vol. 60, no. 11, pp. 3012-3019, 2010.

[16] A. Kahraman and M. Yürüsoy, "Entropy generation due to nonnewtonian fluid flow in annular pipe with relative rotation: constant viscosity case," Journal of Theoretical and Applied Mechanics, vol. 46, no. 1, pp. 69-83, 2008.

[17] D. S. Chauhan and V. Kumar, "Entropy analysis for third-grade fluid flow with temperature-dependent viscosity in annulus partially filled with porous medium," Theoretical and Applied Mechanics, vol. 40, no. 3, pp. 441-464, 2013.

[18] T. Hayat, S. A. Shehzad, and S. Asghar, "MHD flow, thixotropic fluid, thermal radiation, variable thermal conductivity and thermal radiation," Walailak Journal of Science \& Technology, vol. 10, no. 1, pp. 29-42, 2013.

[19] A. M. Siddiqui, M. Hameed, B. M. Siddiqui, and Q. K. Ghori, "Use of Adomian decomposition method in the study of parallel plate flow of a third grade fluid," Communications in Nonlinear Science and Numerical Simulation, vol. 15, no. 9, pp. 2388-2399, 2010.

[20] S. O. Adesanya, "Linear stability analysis of a plane-Poiseuille hydromagnetic flow using Adomian decomposition method," UPB Scientific Bulletin A, vol. 75, no. 2, pp. 99-106, 2013.

[21] S. O. Adesanya, "Steady magnetohydrodynamic visco-elastic heat generating /absorbing slip flow through a porous medium with radiation effect," International Journal of Heat and Technology, vol. 30, no. 1, pp. 69-74, 2012.

[22] S. O. Adesanya and O. D. Makinde, "Heat transfer to magnetohydrodynamic non-newtonian couple stress pulsatile flow between two parallel porous plates," Zeitschrift fur Naturforschung A: Journal of Physical Sciences, vol. 67, no. 10-11, pp. 647-656, 2012 

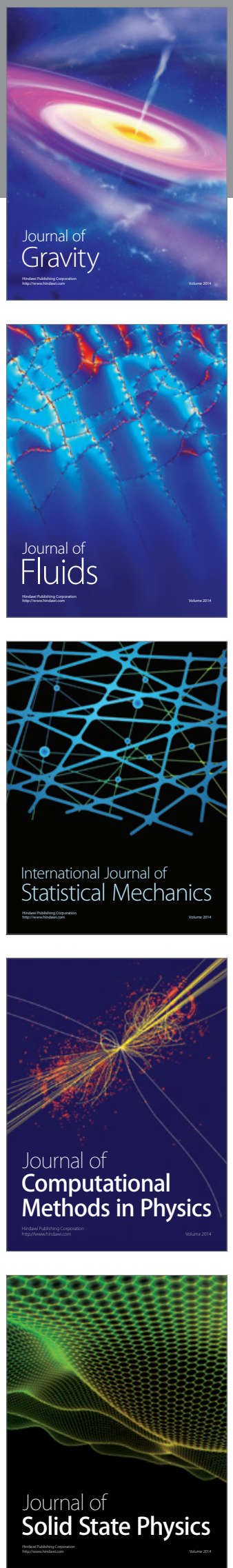

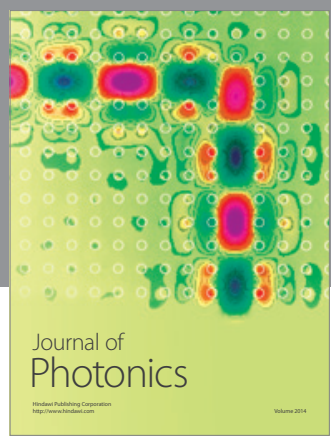

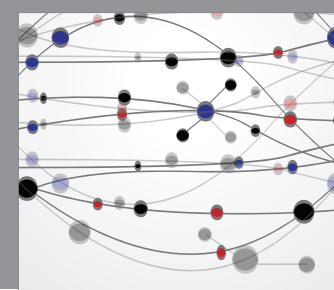

The Scientific World Journal

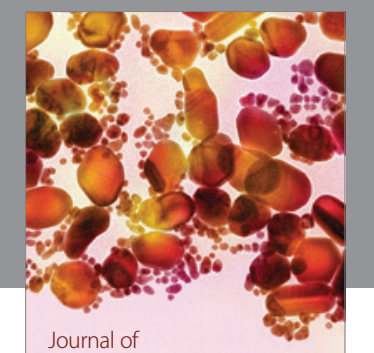

Soft Matter
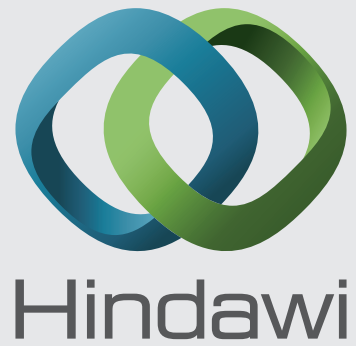

Submit your manuscripts at

http://www.hindawi.com
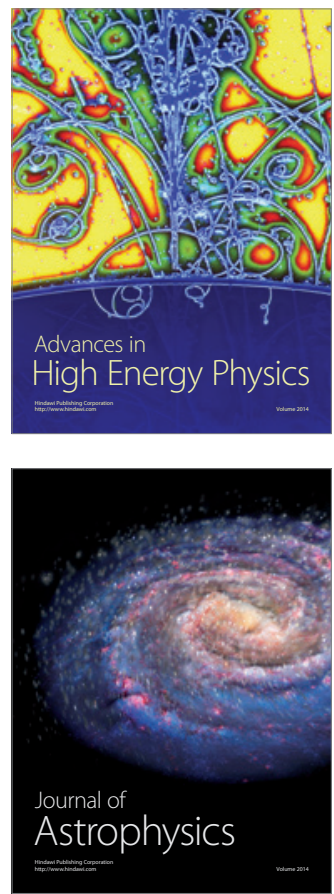
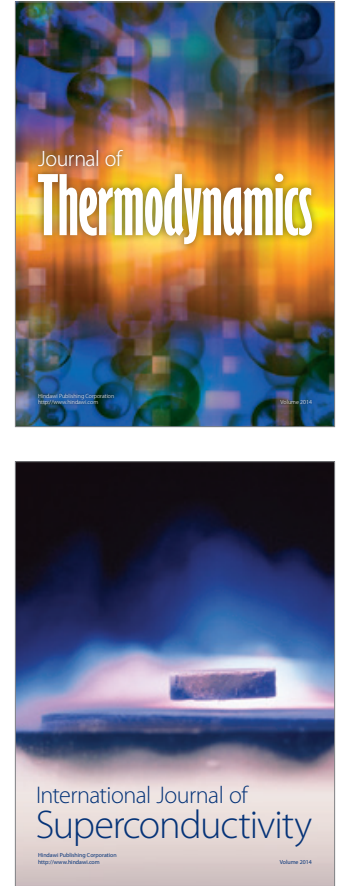
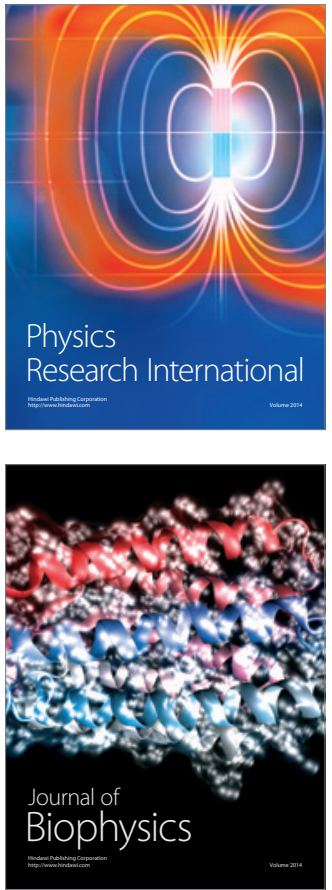
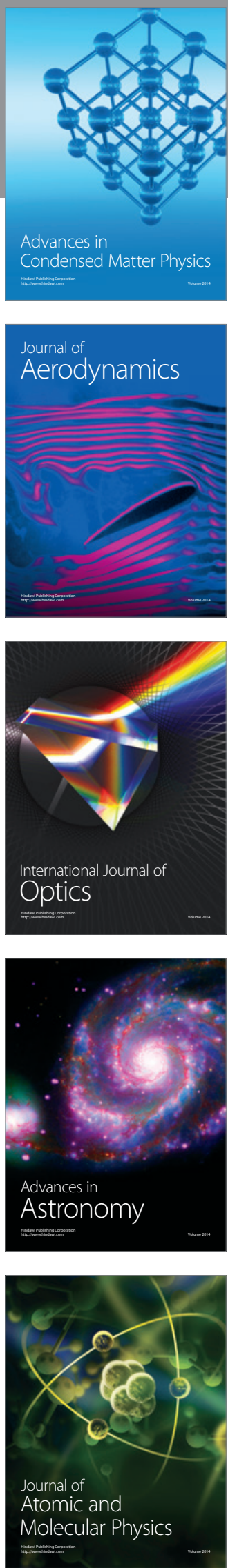